\title{
Vibrations Analysis of Rectangular Plates with Clamped Corners
}

https://doi.org/10.1515/eng-2018-0030

Received September 23, 2017; accepted August 6, 2018

\begin{abstract}
This paper discusses the fundamental natural frequency of a thin elastic rectangular, isotropic and orthotropic, plates with clamped corners. Rayleigh's method was used to analytically calculate the plate lowest natural frequency. In this solution, the vibration mode shape was assumed in a form that certifies the displacement as well as the rotational boundary conditions of the current problem. Finally, this paper provides useful information for evaluating the natural frequency of a plate with fixed corners with different mass attachments configurations.
\end{abstract}

\section{Introduction}

Rectangular elastic plates [1, 2] are widely used in engineering applications; e.g. in printed circuit boards and solar collecting panels. The exact solutions of free vibration of elastic plates are possible only for a few cases, particularly for plate structures with well-defined boundary conditions. However, real-life vibration problems may pose some difficulties in satisfying boundary conditions, such as plates having fixed (or clamped) corners. In these cases, only approximate solutions, using Rayleigh methods, are available [3].

In engineering applications, plates with clamped corners frequently appear in the fatigue life analysis of electronic printed circuit boards (PCBs). For the PCBs, the clamped constraint can arise from the fact that the elastic plate is clamped at each corner using threaded screws, washers and nuts to attach the plate structure to a base support. This type of attachment prevents both displacement and rotation of the structure at the support locations. This kind of problem has been thoroughly investigated us-

\footnotetext{
*Corresponding Author: Mohammad A. Gharaibeh: Department of Mechanical Engineering, the Hashemite University, Zarqa, 13115, Jordan, E-mail: mohammada_fa@hu.edu.jo

Amr M. Obeidat: Department of Electrical Engineering, the

Hashemite University, Zarqa, 13115, Jordan
}

ing the analytical solution of the superposition method invented by Gorman [5-10]. However, this analytical-type solution is very complicated and considered to be impractical.

A similar problem that has been investigated in literature is the vibration of elastic plate with pinned corners. The pinned-corner support boundary condition scheme, unlike the clamped corner scheme, only prevents displacements at the support locations, having no restriction on the rotation. Xiang-sheng [11] used Rayleigh method to analytically approximate the first resonant frequency of a plate having point-pinned supports at plate corners. Kim et al. [12, 13] used beam shape functions to solve for the deflections of plates with point supported at corners. Kerstens [14, 15] used a combination of Rayleigh-Ritz method and modal constraint method to obtain the free vibration problem of plates with such supports. As a powerful tool, the finite element analysis (FEA) has also been extensively used in the vibration analysis of plates with corner supports. Raju et al. [16] and Rao et al. [17] studied the natural frequencies of point-supported elastic plates using finite element method. Pitarresi and Kunz $[18,19]$ developed a simple technique using two-dimensional nonlinear least-squares fit, and finite element simulations, to study the natural frequency of plates having point supports. Recently, Gharaibeh et al. [20, 21] employed the assumed mode method to evaluate the fundamental natural frequency of corner-supported plates using an electronic package.

In addition, elastic rectangular plates having corner supports may exist in many renewable energy and power generation systems such as concentrating solar power (CSP) systems, including solar dishes, parabolic trough solar collectors, and solar central receiver systems. The analysis and characteristics of vibrations of rectangular plates has been widely and extensively investigated based on various techniques. For example, Torabi et al. [22] studied the vibration characteristics of a rectangular plate having a circular central hole with point support, based on the Rayleigh-Ritz method, which is widely used, based on orthogonal polynomials and beam functions. Similarly, Li [23] employed the same approach to characterize vi- 
brations of rectangular plates with general elastic boundary supports. Watkins et al. [24] computed normalized frequencies of elastically point-supported rectangular plates using Eigensensitivity analysis. Shahrjerdi et al. [25] used second-order shear deformation theory (SSDT) to study the vibration of solar-graded plates. Du et al. [26] employed Fourier series method for the in-plane vibration analysis of rectangular plates with elastically restrained edges.

The current work discusses the fundamental natural frequency of an elastic rectangular plate having fixed (or clamped) corners using the Rayleigh method. Both isotropic and orthotropic material systems were considered. In this paper, we first start by introducing the problem of the plate with clamped corners and resulting boundary conditions. This is followed by the use of the Rayleigh method in the system's first natural frequency analysis. Subsequently, the careful selection of the mode shape function that satisfies the displacement and rotational boundary conditions of the problem is also presented. Finally, the natural frequency results for several geometric and mass attachment configurations are discussed in detail.

\section{Description of the Problem}

The plate under consideration in the present work is shown in Figure 1. The supports on the plate corners prevent both displacements and rotations at the support locations, and the edges are completely free elsewhere. The plate width is $(a)$ and length is $(b)$ with thickness $(h)$. The complete set of boundary conditions can be described as:

\section{Displacement}

$$
w(0,0)=w(a, 0)=w(a, b)=w(0, b)=0
$$

\section{Rotations}

$$
\begin{aligned}
& w_{x}(0,0)=w_{x}(a, 0)=w_{x}(a, b)=w_{x}(0, b)=0 \\
& w_{y}(0,0)=w_{y}(a, 0)=w_{y}(a, b)=w_{y}(0, b)=0
\end{aligned}
$$

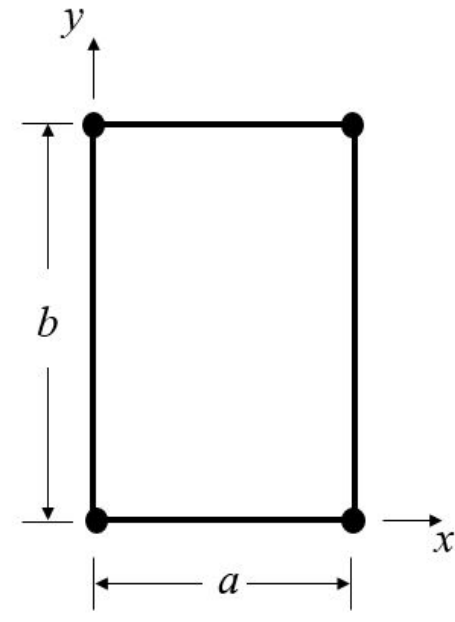

Figure 1: Plate with clamped corners schematic.

\section{Moments}

$$
\begin{aligned}
& w_{x x}(0, y)+v w_{y y}(0, y)=0 \\
& w_{x x}(a, y)+v w_{y y}(a, y)=0 \\
& w_{y y}(x, 0)+v w_{x x}(x, 0)=0 \\
& w_{y y}(x, b)+v w_{x x}(x, b)=0
\end{aligned}
$$

\section{Effective shear forces}

$$
\begin{aligned}
& w_{x x x}(0, y)+(2-v) w_{x y y}(0, y)=0 \\
& w_{x x x}(a, y)+(2-v) w_{x y y}(a, y)=0 \\
& w_{y y y}(x, 0)+(2-v) w_{x x y}(x, 0)=0 \\
& w_{y y y}(x, b)+(2-v) w_{x x y}(x, b)=0
\end{aligned}
$$

\section{Natural Frequency Analysis by Rayleigh's Method}

As previously mentioned, the present work aims to produce an approximate analytical solution for calculating the first natural frequency of a plate with clamped corners using Rayleigh's method. Therefore, it is essential to express the general energy of the system, which is composed 
of the potential and kinetic energies of the plate structure. Considering an isotropic plate having a constant thickness, the plate potential energy $(V)$ can expressed as [27]:

$V=\frac{D}{2} \iint_{A}\left[\left(w_{x x}+w_{y y}\right)^{2}-2(1-v)\left(w_{x x} w_{y y}-w_{x y}^{2}\right)\right] d x d y$

where $D=\frac{E h^{3}}{12(1-v)}$ is the plate flexural rigidity; $h, E$ and $v$ are the plate thickness, plate isotropic material modulus of elasticity, and Poisson's ratio, respectively; $w$ is the plate deflection in the transverse direction; $w_{x x}=\frac{\partial^{2} w}{\partial x^{2}}, w_{y y}=$ $\frac{\partial^{2} w}{\partial y^{2}}$ and $w_{x y}=\frac{\partial^{2} w}{\partial x \partial y}$.

The plate kinetic energy $(T)$ is [27]:

$$
T=\frac{\rho h}{2} \iint_{A}\left[(\dot{w})^{2}\right] d x d y
$$

where $\rho$ is the mass per unit volume of the plate material; $\dot{w}$ is the plate velocity in the lateral direction. The double integrals shown in Eq. (5) and Eq. (6) are evaluated over the plate surface area $A$.

The deflection of the plate, whose motion is harmonic, is:

$$
w(x, y, t)=W(x, y) e^{\hat{i} \omega t}
$$

where $\hat{i}$ is the complex number $\hat{i}=\sqrt{-1}, \omega$ is the vibration circular frequency, and $t$ is the time. The function $W(x, y)$ is the plate mode shape. The proper selection of this mode function will be discussed later.

To use Rayleigh's method, which is based on the energy conservation theorem, the maximum potential energy $\left(V_{\max }\right)$ of the system must be equal to the kinetic energy $\left(T_{\max }\right)$ of the system [8]:

$$
V_{\max }=T_{\max }
$$

where,

$$
\begin{aligned}
& V_{\max }= \\
& \frac{D}{2} \iint_{A}\left[\left(W_{x x}+W_{y y}\right)^{2}-2(1-v)\left(W_{x x} W_{y y}-W_{x y}^{2}\right)\right] d x d y
\end{aligned}
$$

$$
T_{\max }=\omega^{2} \frac{\rho h}{2} \iint_{A}\left[(W)^{2}\right] d x d y
$$

Therefore, the first natural frequency of the plate is written as:

$$
\begin{aligned}
& \omega=\sqrt{\frac{D}{\rho h}} \\
& \sqrt{\frac{\iint_{A}\left[\left(W_{x x}+W_{y y}\right)^{2}-2(1-v)\left(W_{x x} W_{y y}-W_{x y}^{2}\right)\right] d x d y}{\iint_{A}\left[(W)^{2}\right] d x d y}}
\end{aligned}
$$

Considering orthotropic plate material system, the kinetic energy of the plate is still expressed as in Eq. (6) and Eq. (10) above. However, the potential energy is written as [28]:

$$
\begin{aligned}
& V= \\
& \frac{1}{2} \iint_{A}\left[D_{x} w_{x x}^{2}+D_{y} w_{y y}^{2}+2 D_{x} v_{y} w_{x x} w_{y y}+4 D_{k} w_{x y}^{2}\right] d x d y
\end{aligned}
$$

where: $D_{x}=\frac{E_{x} h^{3}}{12\left(1-v_{x} v_{y}\right)}, D_{y}=\frac{E_{y} h^{3}}{12\left(1-v_{x} v_{y}\right)}$ and $D_{k}=$ $\frac{G_{x y} h^{3}}{12}$. The material parameters $E_{x}, E_{y}$ and $G_{x y}$ are the modulus of elasticity in $x$-direction, the modulus of elasticity in $y$-direction, and the shear modulus in $x y$ plane, respectively. Also, $v_{x}$ and $v_{y}$ are Poisson's ratio in $x y$ and $y x$ planes, respectively.

By applying Rayleigh's method as shown previously, the natural frequency of the orthotropic plate can be expressed as:

$$
\omega=\sqrt{\frac{\left[D_{x} W_{x x}^{2}+D_{y} W_{y y}^{2}+2 D_{x} v_{y} W_{x x} W_{y y}+4 D_{k} W_{x y}^{2}\right] d x d y}{\rho h \iint_{A}\left[(W)^{2}\right] d x d y}}
$$

The accuracy of the calculated natural frequency of Eq. (11) and Eq. (13) greatly depends on the engineered selection of the mode shape function, $W(x, y)$, to be discussed next.

\section{Mode Shape Selection}

As explained earlier, the proper selection of the mode shape in the Eq. (11) and Eq. (13) is very important. This mode function should be carefully selected in a mathematical form that satisfies the problem displacement as well as rotational boundary conditions. For the current analysis, the plate is fixed in all directions on the corners and free elsewhere. As formulated in Eq. (1) to Eq. (4), this type of 
constraint will result in zero displacements and rotations at the corners. Whereas the plate deflections and rotations on the edges are different from zero. Therefore, the mode shape of this problem can be formulated as:

$$
\begin{aligned}
& W(x, y)=C_{1}\left(1-\cos \left(\frac{2 \pi x}{a}\right)\right)+C_{2}\left(1-\cos \left(\frac{2 \pi y}{b}\right)\right) \\
& +C_{3}\left(1-\cos \left(\frac{2 \pi x}{a}\right)\right)\left(1-\cos \left(\frac{2 \pi y}{b}\right)\right)
\end{aligned}
$$

where $a$ and $b$ are the plate dimensions in $x$ and $y$ directions, respectively. Also, constants $C_{1}$ and $C_{2}$ are the deflections of the midpoints of the plate sides in $x$ and $y$ directions, respectively. The constant $C_{3}$ represents the displacement of the plate center $\left(x=\frac{a}{2}, y=\frac{b}{2}\right)$. All mode shape constants are independent, as well as unknown, and therefore need to be determined.

Obviously, the mode shape formulated in Eq. (14) completely satisfies the displacement as well as the rotation boundary conditions of Eq. (1) and Eq. (2). However, the satisfaction of the bending moment and shear forces of Eq. (3) and Eq. (4), respectively, are not adequately achieved. Here, if we can satisfy the bending moment and/or effective shear forces boundary conditions, we might be able to greatly increase the calculated natural frequency of Eq. (11) and (13). Furthermore, this can decrease the number of the independent constants of the mode shape function, Eq. (14), therefore achieving much simpler solution. Consequently, we aim to satisfy the shear forces conditions of Eq. (4), having $y=0$ and $x=0$, thus obtaining:

$$
\begin{aligned}
& C_{1}=\beta_{1} C_{3} \\
& C_{2}=\beta_{2} C_{3}
\end{aligned}
$$

where $\beta_{1}$ and $\beta_{2}$ are constants depends on the plate aspect ratio $\left(\frac{b}{a}\right)$, and Poisson's ratio is defined as:

$$
\begin{aligned}
& \beta_{1}=\left(\frac{a}{b}\right)^{2}(2-v) \\
& \beta_{2}=\left(\frac{b}{a}\right)^{2}(2-v)
\end{aligned}
$$

By applying Eq. (15) to Eq. (18), and into Eq. (14), the mode shape function can be modified to:

$$
\begin{aligned}
& W(x, y)=C_{3}\left[\beta_{1}\left(1-\cos \left(\frac{2 \pi x}{a}\right)\right)\right. \\
& +\beta_{2}\left(1-\cos \left(\frac{2 \pi y}{b}\right)\right) \\
& \left.+\left(1-\cos \left(\frac{2 \pi x}{a}\right)\right)\left(1-\cos \left(\frac{2 \pi y}{b}\right)\right)\right]
\end{aligned}
$$

As can be clearly seen in Eq. (19), the number of independent constants in the mode shape are reduced from three to one $\left(C_{3}\right)$. This will result, as expected, in much simpler and accurate solution for the plate with clamped corners problem.

For the orthotropic plate analysis, the shear boundary conditions of the current plate problem become [28]:

$$
\begin{gathered}
w_{x x x}(0, y)+\left(2 \frac{D_{x y}}{D_{x}}-v_{y}\right) w_{x y y}(0, y)=0 \\
w_{x x x}(a, y)+\left(2 \frac{D_{x y}}{D_{x}}-v_{y}\right) w_{x y y}(a, y)=0 \\
w_{y y y}(x, 0)+\left(2 \frac{D_{x y}}{D_{y}}-\frac{D_{x}}{D_{y}} v_{y}\right) w_{x x y}(x, 0)=0 \\
w_{y y y}(x, b)+\left(2 \frac{D_{x y}}{D_{y}}-\frac{D_{x}}{D_{y}} v_{y}\right) w_{x x y}(x, b)=0
\end{gathered}
$$

where $D_{x y}=2 D_{x} v_{y}+2 D_{k}$.

Following the same procedure above, the mode shape constants of Eq. (19) are obtained as:

$$
\begin{aligned}
& \beta_{1}=\left(\frac{a}{b}\right)^{2}\left(\frac{2 D_{x y}-D_{x} v_{y}}{D_{x}}\right) \\
& \beta_{2}=\left(\frac{b}{a}\right)^{2}\left(\frac{2 D_{x y}-D_{x} v_{y}}{D_{y}}\right)
\end{aligned}
$$

\section{Results and Discussions}

\section{Plate with fixed corners with no mass attachments}

To find an equation of the fixed-corners plate's first natural frequency, and considering an isotropic plate system, we substitute Eq. (19), combined with Eq. (17) and Eq. (18), into Eq. (11) to obtain:

$$
\omega=\frac{\lambda^{2}}{a^{2}} \sqrt{\frac{D}{\rho h}}
$$

where $a$ is the plate shortest length, and $\lambda^{2}$ is the plate eigenvalue written as:

$$
\lambda^{2}=4 \pi^{2} \sqrt{\frac{S_{1}+2\left(\frac{a}{b}\right)^{2}+S_{2}\left(\frac{a}{b}\right)^{4}}{S}}
$$

where,

$$
\begin{gathered}
S=6\left(\beta_{1}^{2}+\beta_{2}^{2}\right)+12\left(\beta_{1}+\beta_{2}\right)+8 \beta_{1} \beta_{2}+9 \\
S_{1}=2 \beta_{1}^{2}+4 \beta_{1}+3
\end{gathered}
$$




$$
S_{2}=2 \beta_{2}^{2}+4 \beta_{2}^{2}+3
$$

It important to mention that $\beta_{1}$ and $\beta_{2}$ of Eq. (25) are to be obtained from Eq. (17) and Eq. (18), respectively. As this eigenvalue is a function of $\beta_{1}$ and $\beta_{2}$, then it is a function of plate aspect ratio $b / a$ and Poisson's ratio $v$ only. Assuming that $v=0.3$, then the values of $\beta_{1}, \beta_{2}$ and $\lambda^{2}$ for several plate aspect ratios are summarized in Table 1 .

To simplify the analysis, an empirical formula for calculating the plate eigenvalue as a function of the aspect ratio of the plate has been established. For this formula, the suggested general form is:

$$
\lambda^{2}=a_{1}\left(\frac{1}{\frac{b}{a}}\right)^{a_{2}}
$$

where $a_{1}$ and $a_{2}$ are unknown constants that need to be determined. To determine such constants, a non-linear regression analysis using MINITAB is performed. Thus:

$$
\lambda^{2}=22.27\left(\frac{1}{\frac{b}{a}}\right)^{1.66}
$$

Eq. (27) was compared to the analytical solution data, as shown in Figure 2. From this comparison, it is found that the results of the curve-fitted equation are in agreement with the values of Table 1 .

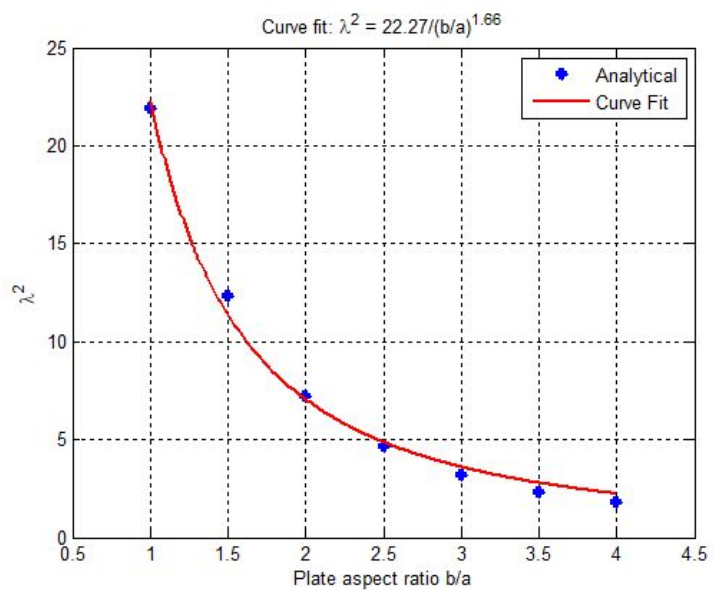

Figure 2: Plate eigenvalues for different plate geometries.

For an orthotropic plate, we substitute Eq. (19), combined with Eq. (21) and Eq. (22), into Eq. (13) to get:

$$
\omega=\frac{4 \pi^{2}}{a^{2}} \sqrt{\frac{D_{x y}}{\rho h}} \sqrt{\frac{S_{x} \frac{D_{x}}{D_{x y}}+2\left(\frac{a}{b}\right)^{2}+S_{y} \frac{D_{y}}{D_{x y}}\left(\frac{a}{b}\right)^{4}}{S_{x y}}}
$$

where:

$$
S_{x y}=6\left(\beta_{1}^{2}+\beta_{2}^{2}\right)+12\left(\beta_{1}+\beta_{2}\right)+8 \beta_{1} \beta_{2}+9
$$

$$
\begin{aligned}
& S_{X}=2 \beta_{1}^{2}+4 \beta_{1}+3 \\
& S_{y}=2 \beta_{2}^{2}+4 \beta_{2}^{2}+3
\end{aligned}
$$

It important to mention that $\beta_{1}$ and $\beta_{2}$ of Eq. (29) are to be obtained from Eq. (21) and Eq. (22), respectively. This eigenvalue is a function of $a / b, E_{x}, E_{y}, v_{x}$ and $v_{y}$.

\section{Plate with fixed corners having mass attachments}

After discussing the problem of a plate with fixed corners, we have a look at the problem of a plate with two mass attachment types:

- Case 1: Plate with fixed corners with a centrally placed mass.

- Case 2: Plate with fixed corners with edge-centerplaced masses.

In the present analysis, only the isotropic plate material system was studied. The same procedure could be followed for orthotropic plate system.

For case 1 , the concentrated mass $(M)$ is mounted at the plate center point $\left(x=\frac{a}{2}, y=\frac{b}{2}\right)$, as shown in Figure 3(a). For case 2, as depicted in Figure 3(b), four equal masses (the mass of each is $M)$ are attached at the centers of the plate edges $\left\{\left(x=\frac{a}{2}, y=0\right),\left(x=a, y=\frac{b}{2}\right),\left(x=\frac{a}{2}, y=b\right)\right.$, $\left.\left(x=0, y=\frac{b}{2}\right)\right\}$.

This mass addition will have no effect on the structure's potential energy. Generally, however, for a plate with attached mass (either for case 1 or case 2), the kinetic energy of the system could be expressed as:

$$
T=\frac{\rho h}{2} \iint_{A}\left[(\dot{w}(x, y))^{2}\right] d x d y+\frac{M}{2} \sum_{i=1}^{N} \dot{w}^{2}\left(\zeta_{i}, \eta_{i}\right)
$$

where $\dot{w}\left(\zeta_{i}, \eta_{i}\right)$ are the velocities of the plate at the mass fix-up locations, and $N$ is the total number of attached masses.

Following the previously described natural frequency analysis procedure, the system's fundamental natural frequency is:

$$
\omega=\frac{\lambda^{2}}{a^{2}} \sqrt{\frac{D}{\alpha_{\frac{M}{a^{2}}}+\rho h}}
$$

where $\alpha$ is the reduced coefficient of the mass, and it is function of plate aspect ratio $b / a$, and Poisson's ratio $v$. Again, assuming that $v=0.3$, then the values of $\alpha$ for several plate aspect ratios, for both cases, are summarized in Table 2.

It is important to mention at this point that this mass addition has no effect on the mode shape constants and 
Table 1: Mode shape constants and isotropic plate eigenvalues for different plate aspect ratios $(v=0.3)$.

\begin{tabular}{cccc}
\hline$b / a$ & $\beta_{1}$ & $\beta_{2}$ & $\lambda^{2}$ \\
\hline 1.0 & 1.700 & 1.700 & 21.916 \\
1.5 & 0.756 & 3.825 & 12.350 \\
2.0 & 0.425 & 6.800 & 7.2033 \\
2.5 & 0.272 & 10.63 & 4.6175 \\
3.0 & 0.189 & 15.30 & 3.1940 \\
3.5 & 0.139 & 20.80 & 2.3370 \\
4.0 & 0.106 & 27.20 & 1.7831 \\
\hline
\end{tabular}

Table 2: Reduced coeficient of the mass for several plate aspect ratios for plate with center mass $v=0.3$ ).

\begin{tabular}{cccccccc}
\hline$b / a$ & $\mathbf{1 . 0}$ & $\mathbf{1 . 5}$ & $\mathbf{2 . 0}$ & $\mathbf{2 . 5}$ & $\mathbf{3 . 0}$ & $\mathbf{3 . 5}$ & $\mathbf{4 . 0}$ \\
\hline$\alpha$ Case 1 & 4.336 & 2.591 & 1.713 & 1.267 & 1.005 & 0.835 & 0.716 \\
$\alpha$ Case 2 & 6.876 & 5.021 & 4.204 & 3.616 & 3.154 & 2.785 & 2.486 \\
\hline
\end{tabular}
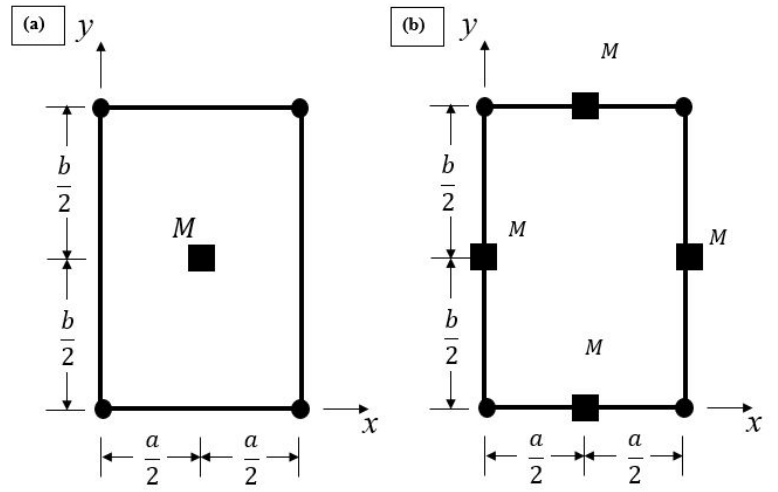

Figure 3: Corner-clamped plate with mass attachments; (a) case 1, (b) case 2 .

plate eigenvalues, and their values are still equal to those listed in Table 1.

As done previously, a general equation to calculate the reduced coefficient of the mass, for both cases, was formulated. The suggested general form for this equation is:

$$
\alpha=c_{1}\left(\frac{1}{\frac{b}{a}}\right)^{c_{2}}
$$

where $c_{1}$ and $c_{2}$ are coefficients determined using a nonlinear regression analysis as $c_{1}=4.35, c_{2}=1.32$ for case 1 , and $c_{1}=6.85, c_{2}=0.72$ for case 2 . A comparison between the fitted formula and the actual (analytical) data of $\alpha$ is shown in Figure 4. For both cases, the fitted formulas are in excellent match with the analytically derived data.

As a special configuration, we shall consider the mass $(M)$ of the added mass (or masses) to be a function of the plate mass. Thus:

$$
M=n b a h \rho
$$

where $n$ is the plate mass fraction, and it can be any positive number: i.e., if $n=0.5$ or $n=2$, then the total added mass is half of the plate mass or double of it, respectively. Now, substitute Eq. (33) into Eq. (31), and after some simple mathematical manipulation we achieve:

$$
\omega=\frac{\lambda^{2}}{a^{2}} \sqrt{\frac{1}{n \alpha \frac{b}{a}+1}} \sqrt{\frac{D}{\rho h}}
$$

or:

$$
\omega=\frac{\Lambda^{2}}{a^{2}} \sqrt{\frac{D}{\rho h}}
$$

where $\Lambda^{2}=\lambda^{2} \sqrt{\frac{1}{n \alpha \frac{b}{a}+1}}$ is the massed plate eigenvalue.

From Eq. (35) above, $\Lambda^{2}$ is now function of $b / a, v$ and $\alpha$, as well as $n$. Luckily, $\alpha$ is function of $b / a$ (besides to $v$ ), for both cases. Therefore, we can implicitly say that $\Lambda^{2}$ is a function of $b / a, v$ and $n$ only. As before, assuming $v=0.3$, Table 3 and Table 4 summarize the values of $\Lambda^{2}$ for different $b / a$ and $n$ combinations for case 1 and case 2, respectively.

To establish a formula to calculate $\Lambda^{2}$ as a function of the plate aspect ratio and of mass fraction factor, for both cases, a general non-linear form, which is a combination of Eq. (27) and Eq. (35), was selected as follows:

$$
\Lambda^{2}=a_{1}\left(\frac{1}{\frac{b}{a}}\right)^{a_{2}} \sqrt{\frac{1}{d_{1} n\left(\frac{1}{\frac{b}{a}}\right)^{d_{2}}+1}}
$$



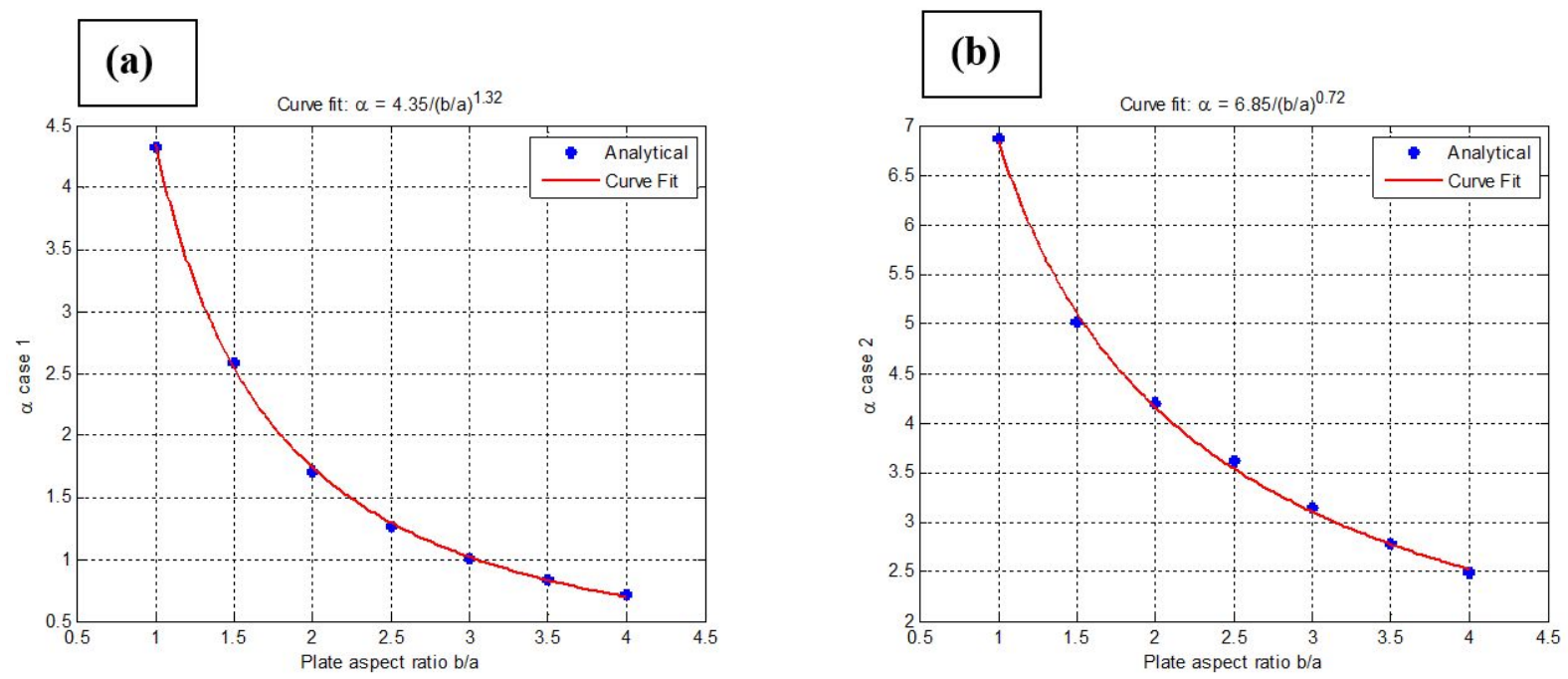

Figure 4: The reduced coefficient of the mass vs plate aspect ratio for (a) case 1 and (b) case 2.
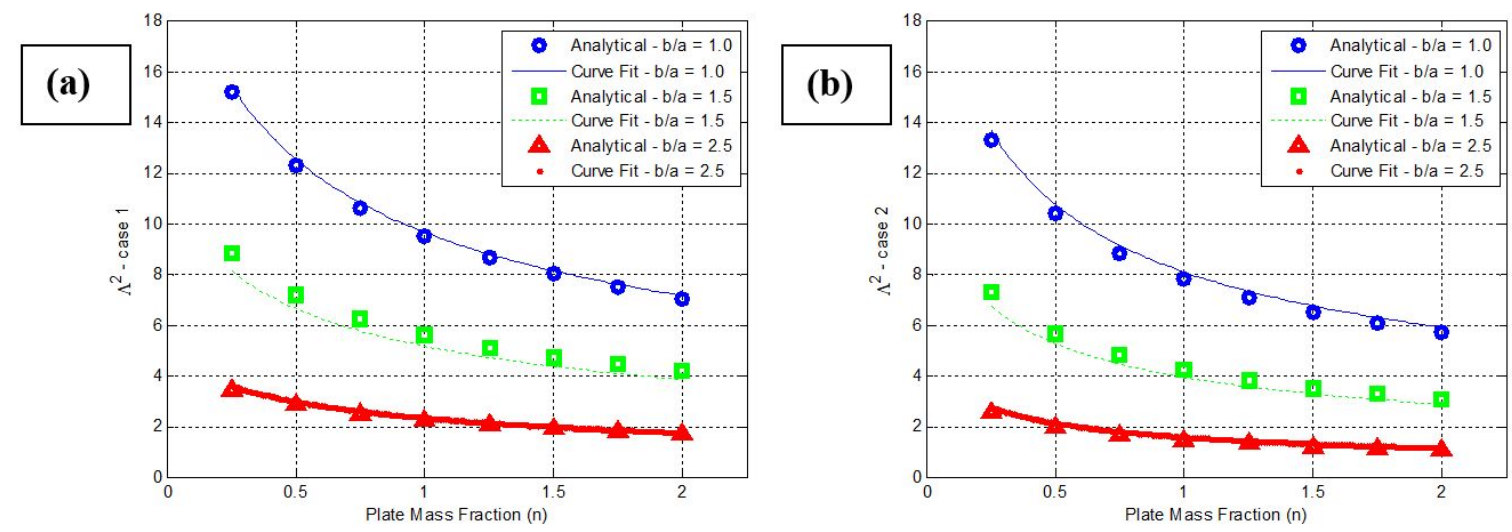

Figure 5: The massed plate eigenvalues $\left(\Lambda^{2}\right)$ vs. the plate mass fraction ( $n$ ) at different $b / a$ for (a) case 1 and (b) case 2.

Here, $a_{1}$ and $a_{2}$, for case 1 and case 2, are similar to those of Eq. (27). Also, $d_{1}$ and $d_{2}$ are two unknown coefficients. To obtain them, a multi-parametric fit was performed considering both case 1 and case 2 . The resultant constant values are $\left(d_{1}=4.33, d_{2}=0.29\right)$ and $\left(d_{1}=\right.$ $\left.6.89, d_{2}=-0.26\right)$ for case 1 and case 2 , respectively. Figure 5 shows a comparison between the analytically derived data and the fitted formulas for both mass attachment cases, for several plate aspect ratios. In conclusion, the $\Lambda^{2}$ values for case 1 and case 2, from both solutions, are in a great agreement.

\section{Conclusions}

This paper has discussed the free vibration problem of plates with clamped corners. Rayleigh's method was used to analytically approximate the fundamental natural frequency of the plate under study. From this approximation, the plate eigenvalue was obtained for several plate dimensions. In addition, two types of mass attachments, namely plate with centrally placed mass and plate with masses on the edges centers, were discussed. A special configuration considering the mass of the added masses as a function of the plate mass was also presented. Finally, this paper developed non-linear equations for calculating the plate eigenvalues for both massed and un-massed configurations. 
Table 3: Values of $\Lambda^{2}$ at different $b / a$ and $n$ for case $1(\nu=0.3)$.

\begin{tabular}{ccccccccc}
\hline \multirow{2}{*}{$b / a$} & \multicolumn{7}{c}{$n$} \\
\cline { 2 - 8 } & 0.25 & 0.50 & 0.75 & 1.0 & 1.25 & 1.50 & 1.75 & 2.0 \\
\hline $\mathbf{1 . 0}$ & 15.1814 & 12.3131 & 10.6283 & 9.4875 & 8.6496 & 8.0005 & 7.4785 & 7.047 \\
$\mathbf{1 . 5}$ & 8.7954 & 7.1987 & 6.2418 & 5.5869 & 5.1026 & 4.7257 & 4.4216 & 4.1696 \\
$\mathbf{2 . 0}$ & 5.2867 & 4.3733 & 3.8127 & 3.4239 & 3.1341 & 2.9073 & 2.7235 & 2.5706 \\
$\mathbf{2 . 5}$ & 3.4495 & 2.8726 & 2.5132 & 2.2619 & 2.0734 & 1.9254 & 1.8052 & 1.7049 \\
$\mathbf{3 . 0}$ & 2.4119 & 2.017 & 1.7687 & 1.594 & 1.4626 & 1.3591 & 1.2749 & 1.2046 \\
$\mathbf{3 . 5}$ & 1.7765 & 1.4896 & 1.3081 & 1.18 & 1.0834 & 1.0072 & 0.9451 & 0.8932 \\
$\mathbf{4 . 0}$ & 1.3612 & 1.1434 & 1.005 & 0.9071 & 0.8332 & 0.7748 & 0.7272 & 0.6874 \\
\hline
\end{tabular}

Table 4: Values of $\Lambda^{2}$ at different $\mathrm{b} / \mathrm{a}$ and $\mathrm{n}$ for case $2(v=0.3)$.

\begin{tabular}{ccccccccc}
\hline \multirow{2}{*}{$b / a$} & \multicolumn{7}{c}{$n$} \\
\cline { 2 - 8 } & 0.25 & 0.50 & 0.75 & 1.0 & 1.25 & 1.50 & 1.75 & 2.0 \\
\hline $\mathbf{1 . 0}$ & 13.291 & 10.4032 & 8.8324 & 7.8092 & 7.0752 & 6.5156 & 6.0707 & 5.7061 \\
$\mathbf{1 . 5}$ & 7.2737 & 5.6572 & 4.7896 & 4.2282 & 3.8269 & 3.5218 & 3.2796 & 3.0814 \\
$\mathbf{2 . 0}$ & 4.0899 & 3.1576 & 2.665 & 2.3485 & 2.1232 & 1.9524 & 1.8171 & 1.7066 \\
$\mathbf{2 . 5}$ & 2.5574 & 1.9653 & 1.6555 & 1.4573 & 1.3166 & 1.2101 & 1.1259 & 1.0571 \\
$\mathbf{3 . 0}$ & 1.741 & 1.3342 & 1.1225 & 0.9875 & 0.8918 & 0.8194 & 0.7622 & 0.7156 \\
$\mathbf{3 . 5}$ & 1.2606 & 0.9643 & 0.8107 & 0.7129 & 0.6436 & 0.5913 & 0.5499 & 0.5162 \\
$\mathbf{4 . 0}$ & 0.955 & 0.7297 & 0.6131 & 0.5390 & 0.4866 & 0.4469 & 0.4157 & 0.3901 \\
\hline
\end{tabular}

\section{References}

[1] Leissa, A. W., Vibration of plates, 1969, NASA SP-160.

[2] Leissa, A. W., The free vibration of rectangular plates, Journal of Sound and Vibration, 1973, 31(3), 257-293.

[3] Rayleigh, J. W. S. B., The theory of sound, 1896, (Vol. 2). Macmillan.

[4] Steinberg, D. S., Vibration analysis for electronic equipment, Second edition, 2000, John Wiley \& Sons.

[5] Gorman, D. J., Free vibration of orthotropic cantilever plates with point supports, Journal of Engineering Mechanics, 1995, 121(8), 851-857.

[6] Singhal, R. K., and Gorman, D. J., Free vibration of partially clamped rectangular plates with and without rigid point supports, Journal of Sound and Vibration, 1997, 203(2), 181-192.

[7] Gorman, D. J., Accurate free vibration analysis of point supported Mindlin plates by the superposition method, Journal of Sound and Vibration, 1999, 219(2), 265-277.

[8] Gorman, D. J., and Singal, R. K., Analytical and experimental study of vibrating rectangular plates on rigid point supports, AIAA Journal, 1991, 29(5), 838-844.

[9] Gorman, D. J., A general analytical solution for free vibration of rectangular plates resting on fixed supports and with attached masses, Journal of Electronic Packaging, 1992, 114, 239.

[10] Gorman, D. J., Vibration analysis of plates by the superposition method, 1999, (Vol. 3), World Scientific.

[11] Xiang-sheng, C., Vibrations of rectangular plates supported at corner points, Applied Mathematics and Mechanics, 1989, 10(8), 751-757.
[12] Kim, C. S., and Dickinson, S. M., The flexural vibration of rectangular plates with point supports, Journal of Sound and Vibration, 1987, 117(2), 249-261.

[13] Kim, C. S., Young, P. G., and Dickinson, S. M., On the flexural vibration of rectangular plates approached by using simple polynomials in the Rayleigh-Ritz method, Journal of Sound and Vibration, 1990, 143(3), 379-394.

[14] Kerstens, J. G. M., Vibration of a rectangular plate supported at an arbitrary number of points, Journal of Sound and Vibration, 1997, 65(4), 493-504.

[15] Kerstens, J. G. M., Vibration of complex structures: the modal constraint method, Journal of Sound and Vibration, 1981, 76(4), 467-480.

[16] Raju, I. S., and Amba-Rao, C. L., Free vibrations of a square plate symmetrically supported at four points on the diagonals, Journal of Sound and Vibration, 1983, 90(2), 291-297.

[17] Rao, G. V., Raju, I. S., and Amba-Rao, C. L., Vibrations of point supported plates, Journal of Sound and Vibration, 1973, 29(3), 387-391.

[18] Pitarresi, J. M., and Kunz, R. J., A simple technique for the rapid estimation of the optimal support locations for a vibrating plate, Journal of Vibration and Acoustics, 1992, 114(1), 112-118.

[19] Kunz, R. J., and Pitarresi, J. M., Analytical and Experimental Optimization of Support Locations for Vibrating Printed Circuit Boards," In Ninth Annual International Electronics Packaging Conference, San Diego, California, 1989, (pp. 11-13).

[20] Gharaibeh, M. A., Finite element modeling, characterization and design of electronic packages under vibration, 2015, PhD Dissertation, State University of New York At Binghamton. 
[21] Gharaibeh, M. A., Su, Q. T., and Pitarresi, J. M., Analytical Solution for Electronic Assemblies Under Vibration, Journal of Electronic Packaging, 2016, 138(1), 011003.

[22] Torabi, K. and Azadi, A.R., Vibration analysis for rectangular plate having a circular central hole with point support by Rayleigh-Ritz method, Journal of Solid Mechanics, 2014, 6(1), 28-42.

[23] Li W.L., Vibration analysis of rectangular plates with general elastic boundary supports, Journal of Sound and Vibrations, 2004, 273(3), 619-635.

[24] Watkin, R.J. and Barton, O., Characterizing the vibration of an elastically point supported rectangular plate using eigensensitivity analysis, Thin-Walled Structures, 2009, 48, 327-333.
[25] Shahrjerdi A., Mustafa F., Bayat M., and Majid D.L.A., Free vibration analysis of solar functionally graded plates with temperature-dependent material properties using second order shear deformation theory, Journal of Mechanical Science and Technology, 2011, 25 (9), 2195-2209.

[26] Du J., Li W., Jin G., Yang T., and Liu, Z., An analytical method for the in-plane vibration analysis of rectangular plates with elastically restrained edges, Journal of Sound and Vibrations, 2007, 306, 908-927.

[27] Timoshenko, S., and Woinowsky-Krieger, S., Theory of plates and shells, 1959, Mc-Graw Hill.

[28] Lekhnitskii, S. G., Anisotropic plates (No. FTD-HT-23-608-67), 1957, Foreign Technology Div Wright-Patterson Afb Oh. 